\title{
Thrombosis centres and AVKs monitoring in COVID-19 pandemic
}

\author{
Doris Barcellona $^{1,2}$ (1) Francesco Marongiu ${ }^{1}(\mathbb{D}$
}

Received: 16 May 2020 / Accepted: 8 July 2020 / Published online: 20 July 2020

(c) Società Italiana di Medicina Interna (SIMI) 2020

\begin{abstract}
Vitamin K-Antagonists (VKAs) are the treatment of choice in patients with indications other than atrial fibrillation and venous thromboembolism. Moreover, some patients still assume VKAs refusing to change their therapy when direct oral anticoagulants (DOACs) are properly indicated. The COVID-19 pandemic has completely changed our lives, nullifying inter-personal relationships to avoid contagion, making difficult the VKAs monitoring. We describe the re-organization of our thrombosis centre (TC) as an example on how to face the emergency due to the COVID-19 pandemic. In the first phase, to avoid overcrowding at the TC, we planned to increase the interval time between INRs checks and to encourage blood sampling at home, especially for elderly patients. Moreover, precise scheduled blood sampling was also organized while telephone and email counselling were guaranteed by two doctors of the TC. In the second phase, to reduce the number of patients who daily attended our TC a switch from VKAs to DOACs was carried out, if no contraindications were identified. In the third phase, to protect patients, healthcare staff and hospital from COVID-19 widespread, telemedicine was strengthened. We tried to extend self-testing at home by means of portable coagulometers to as more patients as possible. To avoid patients staying or coming back to the TC an ad hoc web platform for sending the therapeutic dose adjustment and the next scheduled appointment was developed. The TC re-organization allowed us to monitor anticoagulated patients respecting personal isolation and security measures to avoid possible COVID-19 contagion.
\end{abstract}

Keywords Vitamin K antagonists · Direct oral anticoagulants · Thrombosis centres · COVID-19 pandemic

\section{Introduction}

Oral anticoagulants are life-saving treatment for patients affected by cardiovascular disease. Vitamin K Antagonists (VKAs) have been recently almost replaced by Direct Oral Anticoagulants (DOACs) in the treatment of patients affected by atrial fibrillation and venous thromboembolism [1]. However, there are patients who still need treatment with VKAs due to severe kidney failure or other pathological indications such as mechanical heart valves prosthesis or Antiphospholipid Syndrome. Moreover, some patients still assume VKAs refusing to change their therapy when a DOAC is properly indicated or have to be treated again with VKAs because of DOAC failure [2]. In Italy, the Italian Federation of Centres for the diagnosis and the Surveillance of

Doris Barcellona

doris.barcellona@unica.it

1 Department of Medical Science and Public Health, University of Cagliari, Cagliari, Italy

2 SHRO, Temple University, Philadelphia, PA, USA the Antithrombotic therapy (FCSA) is an important national network of Thrombosis Centres (TC) that have a central role in monitoring patients treated with oral anticoagulants offering a high quality therapy in terms of safety and efficacy [3]. VKAs require a periodic clinical and laboratory monitoring of the prothrombin time (PT) in order to maintain the level of anticoagulation within the therapeutic range.

This means that patients have to go to their TC for blood sampling and to wait for INR result, therapy adjustment and the next scheduled appointment. They have to leave home, often to use public transport to reach the TC, stay in overcrowding waiting rooms, exposing themselves to contact with other people. The COVID-19 pandemic [4] has completely changed our lives, nullifying inter-personal relationships to avoid contagion. At this time, it may be useful to re-organize the daily clinical practice in a TC whose task is to monitor especially elderly frail patients, at high risk of contagion and death. 


\section{A model of thrombosis centre re-organization: our experience}

In this report, we describe the re-organization of our $\mathrm{TC}$ that could be an example on how to face the emergency due to the COVID-19 human-to-human infection (Table 1).

Our TC belong to the FCSA and a total of 1110 patients are followed for their oral anticoagulant therapy.

COVID-19 infection has completely changed our organisation forcing medical staff to work hard during the first period of the pandemic to avoid overcrowding and close contact by patients who attend the TC for monitoring their therapy.

\section{How to avoid overcrowding at the thrombosis center?}

On March 11, the World Health Organization declared the COVID-19 pandemic and in Italy the lockdown began.

In the first phase, all patients were called on phone in order to plan blood samples in groups of 6 people every 30 min. Because of COVID-19, the time to take blood sample has lengthened. In our hospital, before attending the TC, patients have to fill out a questionnaire about their state of health, contacts with people at risk of infection or affected by COVID-19 and any movements from their home. Filling out the questionnaire followed by a careful patients' hands washing with hydroalcoholic solutions take at least $10 \mathrm{~min}$. Moreover, for each patient, nurses have to change gloves and disinfect the surface where they have made blood sampling.

Moreover, patients were advised either to wear a face mask to prevent the COVID-19 contagion or to stay home and call their general practitioner in case of fever, dry cough and sore throat. Our teaching hospital is a COVID-19 free hospital as

Table 1 Thrombosis centre re-organization

First phase
How to avoid overcrowding
Blood sampling precisely scheduled
Reduction of frequency of INR monitoring
Blood sampling at home
Telephone and e-mail couselling
Second phase
How to reduce patients' attending TC
Switch from AVKs to DOACs whenever possible
Third phase
Telemedicine
Self-testing at home
Web platform where to send INR results and AVKs dosages

the Local Health System decided, so we are not allowed to admit this kind of patients.

It is known that the mortality rate for COVID-19 is higher in elderly patients affected by cardiovascular and chronic respiratory disease, diabetes and obesity [5], pathological conditions that are frequently found in anticoagulated patients. Most of our patients over the age of 80 , who cannot be treated with DOAC, are therefore advised to carry out their blood sampling at home. In our region home blood sampling is arranged by the Local Health System only for patients who have obtained home care because of their comorbidities, otherwise all patients have to pay a nurse.

All INRs are performed in our Thrombosis and Hemostasis Departmental Unit laboratory while INRs carried out in other laboratory are not accepted. This attitude is intended to avoid the possible inter-laboratory INR variability and therefore to preserve the effectiveness and safety of the VKAs dosage. Our laboratory performs two types of quality control: internal and external, the latter is organized by the FCSA once a year by sending to the laboratory unknown INR lyophilized plasmas.

The frequency of the INR monitoring in our TC has been reduced. Before this pandemic period, it used to be as follows [6]: if the INR value was stable within the therapeutic range the next check was booked after 4 weeks, if it was out of range the VKAs dosage was changed and patient was ask to return after 10 days. A more strictly control (1, 2 days) was used when a patient was in an overdose state. Right now, the time interval between one INR check and the other has been significantly increased: from a minimum of 5-6 days in case of overdose to a maximum of 6 weeks when the level of anticoagulation is stable [7].

The median percentage of time spent in the therapeutic range during the last 3 months (March-May) was not different from that in the previous three ones (December-February): $81 \%,(8-100 \%)$ in both periods. No bleeding or thrombotic events were recorded.

Although, COVID-19 infection is not as widespread as in other Italian region, anticoagulated patients often referred to our TC for having information about the risk of getting COVID-19, the correct way to use the individual protection devices and a delay in their scheduled appointment for fear of going to the hospital and getting the infection. Two of the doctors at the TC worked every day answering the phone calls and e-mails sent us by the patients, reassuring them and providing accurate and detailed informations.

\section{How to reduce the number of patients which daily attended to our thrombosis centre?}

In the second phase, the clinical history of all patients has been reviewed to identify the patients that could be switched from VKAs to DOACs. It is well known that DOACs therapy 
does not require a closed monitoring [1]; therefore, it is the best treatment for patients who have the indication, in a condition where the lockdown is life-saving as the anticoagulant therapy. Moreover, in Italy, DOACs are reimbursed by the National Health System.

Before COVID-19 pandemic, most patients $(n=519$, $47 \%$ ) were on VKAs therapy. All the naïve patients affected by atrial fibrillation and venous thromboembolism are treated with DOACs if no contraindications do exist, but the most difficult task is the transition from VKAs to DOACs in non-naïve patients already on VKAs therapy.

Several patients preferred to continue monitoring their PT and looked very wary at the new drugs despite being provided with precise informations about the risks and benefits of both medications [8]. Especially very elderly patients, who have been treated with VKAs for many years and have never had adverse events such as bleeding or thrombosis, refused to take DOACs probably because they were more wary of novelty and less conducive to changes in their healthy lifestyle.

At the moment, after almost 2 months, in our Thrombosis Centre, the number of patients treated with DOACs is increased by $20 \%$ reaching a total number of 691 . Many patients, aware of the danger of contagion and the need to comply with the lockdown, have eventually agreed to change their therapy, putting aside their anxieties and doubts.

\section{How to improve the thrombosis centre re-organization?}

In the third phase, we addressed the problem of those patients who did not have an indication to DOACs or who did not want to take them. The number of patients still treated with VKAs were 418.

Portable coagulometers can provide significant aid in the management of the VKAs treatment since it allows patients to perform the PT INR at home [9]. Portable coagulometers has been evaluated in several randomized clinical trials and meta-analysis $[10,11]$ showing that their use is associated with a reduction of thromboembolic events and similar safety when compared to standard care.

In our thrombosis centre, portable coagulometers have been implemented since 2004 and 110 patients are monitored in self-testing at home [12]. An ad hoc web platform allows patients to send the INR result via web and to communicate with the doctors at the TC by means of a questionnaire about possible adverse events, changes in medications, scheduled surgery and proper drug assumption. In the same way, patients receive the dosage adjustment and the next scheduled appointment sent back by the doctors of the TC. Good results in terms of safety, efficacy, and patients' satisfaction were obtained. VKAs monitoring by self-testing at home improves adherence to the therapy and patients' quality of life, especially in elderly who can avoid to attend the TC. This advantages are counterbalanced by the high cost of the device and the test strips.

Only one patient, in the COVID-19 period, accepted to buy the portable coagulometer and he attended the educational course, held by our biologist, through a video phone call.

Because of this problem, 307 patients needed to be monitored at our TC. The COVID-19 pandemic gave us an opportunity to further develop telemedicine. To avoid patients from waiting or having to return to the TC to withdraw the PT INR result a new ad hoc web platform has been created. It is linked with our software for managing anticoagulated patients (TAOnet, EDP-Progetti, Bolzano, Italy), that allows doctors to send the therapy on the web by a click. The patients can log in with a personal ID and password to view and print their VKAs dosage scheme and the next scheduled appointment without leaving their home.

We are also trying to further improve our telemedicine approach to the monitoring of oral anticoagulants. Patients, especially those being treated with DOACs, will allowed to upload on the platform their hematochemical examinations and reports of other specialists that could be sent to the TC via web. A questionnaire similar to that administered to VKAs patients will also be available for DOACs patients.

\section{Conclusions}

The TC re-organization allowed us to monitor anticoagulated patients respecting personal isolation and security measures to avoid possible COVID-19 contagion. None of our patients suffered from COVID-19 infection.

Overcrowding at the TC is avoided by precise scheduled blood sampling, increasing the interval time between INRs checks and encouraging blood sampling at home. Telephone and email counselling were guaranteed by doctors of the TC.

To reduce the number of patients who attend our TC, VKAs have been replaced by DOACs, whenever possible.

Telemedicine help us to avoid patients from attending, waiting or having to come back to the TC to withdraw the PT INR result and the therapeutic dose adjustment. However, the need to expand the use of the portable devices to as many patients as possible is countered by the costs that patients have to afford purchasing the INR point-of-care and the reactive strips. The lockdown on one hand has improved the COVID-19 pandemic limiting the contagions, but on the other has worsened the economic conditions of many people who cannot buy this device. In Italy, the high cost of the portable coagulometer and the reactive strips is in borne by the patients. An appeal to health authorities and industries for the reduction of this economic burden should be made. 
It remains the sadness of not being able to shake hands and to receive a hug, even if the expression of the eyes, the only part of the face that the face mask does not cover, has become the new manifestation of affection, esteem and mutual trust.

\section{Compliance with ethical standards}

Conflict of interest The authors have no conflict of interest to declare.

Statement of human and animal rights This article does not contain any studies with human participants or animals performed by any of the authors.

Informed consent The patients informed consent is not applicable in this case since the manuscript describe the general organization of the $\mathrm{TC}$ without using patient's data.

\section{References}

1. Prisco D, Ageno W, Becattini $C$ et al (2017) Italian intersociety consensus on DOAC use in internal medicine. Intern Emerg Med 12:387-406

2. Kajy M, Mathew A, Ramappa P (2019) Treatment failures of direct oral anticoagulants. Am J Ther. https://doi.org/10.1097/ MJT.0000000000001083

3. Palareti G, Antonucci E, Migliaccio L et al (2017) Vitamin K antagonist therapy: changes in the treated populations and in management results in Italian anticoagulations clinics compared with those recorded 20 years ago. Intern Emerg Med 12:1109-1119

4. Rothan HA, Byrareddy SN (2020) The epidemiology and pathogenesis of coronavirus disease (COVID-19) outbreak. J Autoimmun 109:102433
5. Wu Z, McGoogan JM (2020) Characteristics of and important lessons from the coronavirus disease 2019 (COVID-19) outbreak in China: summary of a report of 72314 cases from the Chinese Center for Disease Control and prevention. JAMA 323:1239-1242

6. Barcellona D, Vannini ML, Fenu L, Balestrieri C, Marongiu F (1998) Warfarin or Acenocoumarol: which is better in the management of oral anticoagulants? Thromb Haemost 80:899-902

7. Barnes GD, Kong X, Cole D, Haymart B, Kline-Rogers E, Almany S, Dahu M, Ekola M, Kaatz S, Kozlowski J, Froehlich JB (2018) Extended international normalized ratio testing intervals for warfarin-treated patients. J Thromb Haemost 16:1307-1312

8. Barcellona D, Luzza M, Battino N, Fenu L, Marongiu F (2015) The criteria of the Italian Federation of Thrombosis Centres on DOACs: a "real world" application in nonvalvular atrial fibrillation patients already on vitamin K Antagonist. Intern Emerg Med 10:157-163

9. Barcellona D, Fenu L, Marongiu F (2017) Point of care testing: an overview. Clin Chem Lab Med 55:800-805

10. Bloomfield HE, Krause A, Green N, Taylor BC, MacDonald R, Rutks I, Reddy P, Wilt TJ (2011) Meta-analysis: effect of patient self-testing and self-management of long term anticoagulation on major clinical outcomes. Ann Intern Med 154:472-482

11. Sharma P, Scotland G, Cruickshank M, Tassie E, Fraser C, Burton C, Croal B, Ramsay CR, Brazzelli M (2015) Is self-monitoring an effective option for people receiving long-term vitamin $\mathrm{K}$ antagonist therapy? A systematic review and economic evaluation. BMJ Open 5:e007758

12. Barcellona D, Mastino D, Marongiu F (2018) Portable coagulometer for vitamin $\mathrm{K}$-antagonist monitoring: the patients' point of view. Patient Prefer Adherence 12:1521-1526

Publisher's Note Springer Nature remains neutral with regard to jurisdictional claims in published maps and institutional affiliations. 